\title{
Spacers were better and less expensive than nebulisers for giving albuterol to children with moderate to severe acute asthma
} Leversha AM, Campanella SG, Aickin RP,et al. Costs and effectiveness of spacer versus nebulizer in young children with
moderate and severe acute asthma.J Pediatr 2000 Apr;136:497-502.

QUESTION: In young children seen in the emergency department for moderate and severe acute asthma, is delivery of albuterol by metered dose inhaler (MDI) and spacer a cost effective alternative to delivery by nebuliser?

Design

Randomised (allocation concealed*), blinded \{patients, clinicians, and outcome assessors $\} \nmid{ }^{*}$ controlled trial with 48 hour follow up.

\section{Setting}

A children's hospital in Auckland, New Zealand.

\section{Patients}

60 children who were 1 to 4 years of age (mean age 34 mo, $64 \%$ boys), had a known history of asthma, and presented to the emergency department with an acute exacerbation of asthma that was rated as moderate to severe. Exclusion criteria were inhaled bronchodilator use in the hour before presentation, immediate admission to the intensive care unit, or a co-existing medical condition, such as pneumonia. Follow up was complete.

\section{Intervention}

Children were allocated to albuterol, $600 \mu \mathrm{g}$ by MDI and spacer $(\mathrm{n}=30)$, or to albuterol, $2.5 \mathrm{mg}$ by nebuliser over 10 minutes $(n=30)$. Treatments were repeated as needed at 20 minute intervals (maximum 6 treatments).

\section{Main cost and outcome measures}

The main outcome measures were clinical severity scores, heart rate, respiratory rate, oxygen saturation rate, and wheezing (score 0 [none] to 4 [silent chest]) at 60 minutes; admission to hospital; and visits to the emergency department at 48 hours. Fixed costs, human resources, and investigation and treatment costs were assessed in 1996 New Zealand dollars.

\section{Main results}

Wheezing was reduced more in the spacer group after the first treatment than in the nebuliser group (mean difference in wheeze score $0.45,95 \%$ CI 0.07 to 0.9 , $\mathrm{p}=0.03$ ). The increase in heart rate after first treatment was smaller in the spacer group than in the nebuliser group (mean difference 8.1 beats/min, CI 5.3 to 16.4, $\mathrm{p}<0.01$ ). After adjustment for sex, fewer children in the spacer group than in the nebuliser group required admission to the hospital ( $33 \% v 60 \%, \mathrm{p}=0.04$; number needed to treat 4). Mean total costs were lower for the spacer group than for the nebulizer group (\$825 v $\$ 1282, \mathrm{p}=0.03)$. Groups did not differ for clinical severity score, respiratory rate, oxygen saturation, tremor, or hyperactivity.

\section{Conclusion}

In children seen in the emergency department for moderate and severe asthma, delivery of albuterol by metered dose inhaler and spacer was a cost effective alternative to delivery by nebuliser.

*See glossary.

$\nmid$ Information provided by author.

\section{COMMENTARY}

Evidence has been building on the effectiveness of MDIs and spacers for the delivery of salbutamol to children with acute asthma. ${ }^{12}$ This study by Leversha et al adds to the body of evidence and also provides data on the cost effectiveness of MDIs and spacers relative to nebuliser delivery systems. On the basis of this study and previous randomised trials, I think paediatric emergency departments should adopt MDIs and spacers as the primary delivery system for children presenting with acute asthma.

The $60 \%$ admission rate in the nebuliser group seems to be strikingly high. Most of the cost savings in the spacer group resulted from avoiding admissions. The explanation for this marked difference in admission rates between groups may be the fact that patients with severe disease were enrolled in the trial and that MDIs and spacers are that much more effective than nebulisers. However, in this relatively small trial of 60 patients, one must also consider the role of chance and statistical fluctuations because of the small sample size. Previous research on children did not show a reduced probability of hospital admission but did show that use of MDIs and spacers resulted in a shorter length of stay in the emergency department. ${ }^{2}$

This study used high quality methods (low risk for bias). The investigators ensured allocation concealment, and if the Jadad quality scale were applied, they would score top marks (5 out of 5 ) for this study. ${ }^{3}$

We can therefore be confident that MDIs and spacers are at least as effective as nebulisers for delivery of salbutamol. Given the possibility that they are more cost effective, it makes potential economic sense to adopt MDIs and spacers as our standard of care in paediatric emergency departments.

Terry Klassen, MD University of Alberta Edmonton, Alberta, Canada

1 Amirav I, Newhouse MT. Metered-dose inhaler accessory devices in acute asthma: efficacy and comparison with nebulisers: a literature review. Arch Pediatr Adolesc Med 1997;151:876-82.

2 Cates CJ, Rowe BH. Holding chambers versus nebulisers for beta-agonist treatment of acute asthma. Cochrane Database Syst Rev 2000; latest version Sep 281999.

3 Moher D, Pham B, Jones A, et al. Does quality of reports of randomised trials affect estimates of intervention efficacy reported in meta-analyses? Lancet 1998;352:609-13. 